\title{
Ensembling complex network 'perspectives' for mild cognitive impairment detection with artificial neural networks
}

\author{
Eufemia Lella $^{\mathrm{a}}$, Gennaro Vessio ${ }^{\mathrm{b}, *}$ \\ ${ }^{a}$ Innovation Lab, Exprivia S.p.A., Molfetta, Italy \\ ${ }^{b}$ Department of Computer Science, University of Bari, Bari, Italy
}

\begin{abstract}
In this paper, we propose a novel method for mild cognitive impairment detection based on jointly exploiting the complex network and the neural network paradigm. In particular, the method is based on ensembling different brain structural "perspectives" with artificial neural networks. On one hand, these perspectives are obtained with complex network measures tailored to describe the altered brain connectivity. In turn, the brain reconstruction is obtained by combining diffusion-weighted imaging (DWI) data to tractography algorithms. On the other hand, artificial neural networks provide a means to learn a mapping from topological properties of the brain to the presence or absence of cognitive decline. The effectiveness of the method is studied on a well-known benchmark data set in order to evaluate if it can provide an automatic tool to support the early disease diagnosis. Also, the effects of balancing issues are investigated to further assess the reliability of the complex network approach to DWI data.
\end{abstract}

Keywords: Decision support systems, Mild cognitive impairment, Diffusion-weighted imaging, Complex networks, Artificial neural networks

\section{Introduction}

Mild cognitive impairment (MCI) is a non-disabling disorder characterized by an early state of abnormal cognitive function (Petersen 2016). An individual with MCI shows measurable changes in thinking skills-usually noticed by family members and friends-, which however do not affect the ability to carry out daily life activities. Nevertheless, according to some studies reported in literature, e.g. (Ward et al. 2013; Vos et al., 2015), people with $\mathrm{MCI}$ are at a higher risk of developing Alzheimer's disease (AD), or other kinds of dementia, than people without MCI. Research is ongoing to identify and validate useful biomarkers that might indicate the risk of decline (Reitz and Mayeux. 2014). These may be used to support the early disease diagnosis and testing of novel treatments.

\footnotetext{
${ }^{*}$ Corresponding author

Email address: gennaro.vessio@uniba.it (Gennaro Vessio )
}

Unfortunately, despite the large number of promising results, biological markers of MCI are at various stages of development and their use within standard clinical routines has not yet been established.

Advances in this research have been obtained, in the last few years, in neuroimaging, particularly with diffusion-weighted imaging (DWI). DWI measures the water diffusion along the white matter (WM) fibers, thus it can provide meaningful information regarding their integrity (Amlien and Fjell, 2014). This information can highlight WM micro-structural changes that are related to neurodegeneration. In addition, when combined with tractography algorithms, DWI enables the reconstruction of the WM fiber tracts, providing a characterization of the physical connections of the brain that can be subsequently investigated through a complex network-based approach, e.g. (Lo et al. 2010). In fact, the human brain can be modeled as a network the nodes of which are the anatomical regions, while edges represent the fiber tracts connecting 
them.

A recently proposed approach to study the diagnostic potentials of complex network measures consists in feeding these measures into supervised machine learning algorithms to automatize the disease detection, e.g., (Ebadi et al. 2017, Schouten et al., 2017, Lella et al., 2018). Developing a computerized decision support tool is desirable as it can provide a complementary approach to the standard evaluations which is non-invasive and low-cost. However, while models with very high prediction accuracy in detecting late-stage $\mathrm{AD}$ have been developed so far, the binary discrimination healthy/MCI is still hard. MCI, in fact, seems to be characterized by very minimal variations which makes it difficult to find meaningful patterns for distinguishing this state from normal aging. Furthermore, several existing works on this problem used either private data, e.g. (Wee et al., 2011; Ebadi et al., 2017), or benchmark data with a disproportion of the MCI group compared to the control group, e.g. (Nir et al., 2015; Prasad et al., 2015). In the first case, the experimentation is not reproducible. In the second case, since machine learning algorithms are known to prefer the majority class when data are unbalanced, estimating sensitivity and specificity values may be biased by this factor.

The contribution of this paper is two-fold. On one hand, we propose a novel classification strategy for the binary discrimination healthy/MCI. Starting from the observation that different complex network measures may provide different "perspectives" of the same networks under study, i.e. they carry on non-redundant information, we develop an ensemble model based on artificial neural networks each trained on different complex network features. Ensemble models are typically based on different classifiers which learn to predict the target output based on the same input. In the proposed method, instead, the ensemble is based on copies of the same classifier fed with different inputs. On the other hand, this paper contributes by investigating the effects on classification performance of balancing the two groups under investigation. To this end, some well-known under-sampling approaches are employed. To our best knowledge, this is the first attempt to study the impact of balancing issues to evaluate the reliability of the complex network approach to DWI data.

\section{Materials}

Data used in the preparation of this article were obtained from the Alzheimer's Disease Neuroimaging Initiative (ADNI) database (adni.loni.usc.edu). The ADNI was launched in 2003 as a public-private partnership, led by Principal Investigator Michael W. Weiner, MD. The primary goal of ADNI has been to test whether serial magnetic resonance imaging, positron emission tomography, other biological markers, and clinical and neuropsychological assessment can be combined to measure the progression of MCI and early AD. For up-to-date information, see www. adni-info.org.

In particular, we used an unbalanced cohort of $108 \mathrm{MCI}$ patients and 49 healthy control (HC) subjects. Control subjects did not show signs of depression, MCI or dementia; MCI patients reported a subjective memory concern, but without any significant impairment in other cognitive domains: they preserved daily life activities with no sign of dementia. Scans were acquired using a 3-T GE Medical Systems scanner; more precisely, 46 separate images were acquired for each scan: 5 with negligible diffusion effects ( $b_{0}$ images) and 41 diffusion-weighted images $(b=1000$ $\mathrm{s} / \mathrm{mm}^{2}$ ). For each subject, the $\mathrm{T} 1$ anatomical scan has also been used to perform tractography.

All procedures followed were in accordance with the ethical standards of the responsible committee on human experimentation (institutional and national) and with the Helsinki Declaration of 1975 and its later amendments. The ADNI project was approved by the Institutional Review Boards of all participating institutions and informed consent was obtained from all patients for being included in the study.

\section{Methods}

The analysis we carried out is described in the following subsections.

\subsection{Image Processing}

Image processing consisted in the reconstruction of the brain connectome from the DWI scans. For each subject, the DICOM images were acquired from ADNI and the dcm2nii tool, provided with the MRIcron suite (https: //www.nitrc.org/projects/mricron), was used to 


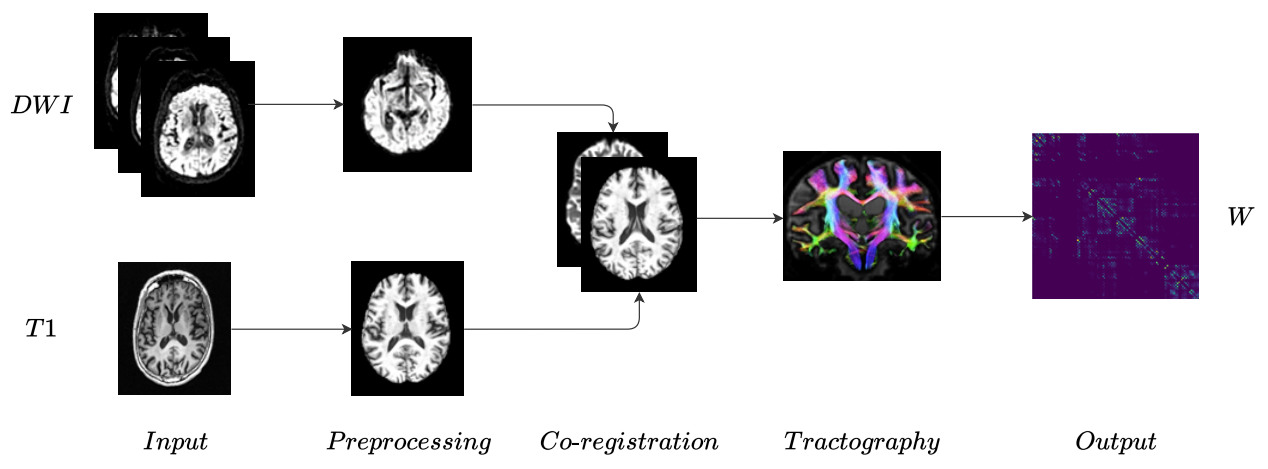

Figure 1: Image processing pipeline. DWI and T1 scans underwent several processing steps to obtain a weight connectivity matrix $W$ as final output (Lella and Estrada 2020).

convert them into the NIFTI format. The NIFTI images were then re-organized in the standard BIDS format.

The subsequent processing steps, from image preprocessing to co-registration and structural connectome generation, were performed using tools provided by the FSL FMRIB Software Library (FSL) (https://fsl. fmrib.ox.ac.uk/fsl/fslwiki) and the MRtrix3 software package (https://www.mrtrix.org/). The main steps, which are well-established in the literature, are shown in Fig. 1] First, a denoising step was performed to enhance the signal-to-noise ratio of the diffusion weighted signals so as to reduce the thermal noise. This noise is due to the stochastic thermal motion of the water molecules and their interaction with the surrounding micro-structure (Veraart et al. 2016). Head motion and eddy current distortions were corrected by aligning the DWI images of each subject to the average $b_{0}$ image. Then, the brain extraction tool (BET) was used for the skull-stripping of the brain (Smith, 2002). The bias-field correction was used to correct all DWI volumes. Similarly, the T1 weighted scans were processed by performing the following steps: reorientation to the standard image MNI152, automatic cropping, bias-field correction, registration to the linear and nonlinear standard space and brain extraction. The next step was the inter-modal registration of the diffusionweighted and $\mathrm{T} 1$ weighted images.

After preprocessing and co-registration, the structural connectome was generated. First, we generated a tissuesegmented image tailored to the anatomically constrained tractography (Zhang et al. 2001). Then, we performed an unsupervised estimation of WM, gray matter and cerebro- spinal fluid. In the next step, the fiber orientation distributions for spherical deconvolution was estimated (Jeurissen et al. 2014). We then performed a probabilistic tractography (Tournier et al. 2010) using dynamic seeding (Smith et al., 2015b) and anatomically-constrained tractography (Smith et al., 2012), which improves the tractography reconstruction by using anatomical information through a dynamic thresholding strategy. We applied the spherical-deconvolution informed filtering of tractograms (SIFT2) methodology (Smith et al. 2015b), which not only provides more biologically meaningful estimates of the structural connection density, but also a more efficient quantification of the streamlines connectivity. The obtained streamlines were mapped through a T1 parcellation scheme by using the AAL2 atlas (Rolls et al., 2015), which is a revised version of the automated anatomical atlas (AAL) including 120 regions. Finally, a robust structural connectome construction was performed for generating the connectivity matrices (Smith et al. 2015a). The pipeline here described has also been used in recent structural connectivity studies, for example (Amico and Goñi 2018) and (Tipnis et al., 2018).

The final output was a $120 \times 120$ weighted symmetric connectivity matrix $W$ for each subject, the entries $w_{i j}$ of which corresponded to the number of fiber tracts connecting the anatomical region $i$ to region $j$ in accordance with the AAL2 atlas.

\section{Complex Network Features}

The connectivity matrix $W$ represents the structural complexity of the brain. From $W$ several graph measures 
can be computed to describe its topological properties. In this work, we consider three measures, namely the original weight information resulting from the application of tractography, shortest path length and weighted communicability. For each node pair $i j$, the shortest path length is simply the length of the shortest path from $i$ to $j$. It provides a different information from weights as it expresses the efficiency of the information flow. Analogously, communicability, firstly introduced by Estrada and Hatano (Estrada and Hatano, 2008), then refined by Crofts and Higham (Crofts and Higham, 2009) in the weighted case, is defined as:

$$
C_{i j}=\left(\exp \left(D^{-1 / 2} W D^{-1 / 2}\right)\right)_{i j},
$$

where $D \in \mathbb{R}^{N \times N}$ is the diagonal strength matrix with $N$ the number of nodes. This network metric provides an even more general measure of the ease of communication inside the network, as it takes into account not only the shortest paths but all available routes connecting two nodes. Its usefulness in assessing the altered brain connectivity due to $\mathrm{AD}$ has been observed in recent works (Lella et al., 2018, 2019).

\subsection{Model Fitting}

Similar or conceptually different classification models can be combined through a voting scheme, so that the individual weaknesses of each classifier are mitigated (Theodoridis, 2015). Different models trained independently, in fact, can look at slightly different aspects of the data, so having a chance to improve the predictions provided by each individual model in the ensemble. In particular, since different complex network measures are likely to provide different "perspectives" of the same networks under study, i.e. they carry on non-redundant information, the ensemble of these features can take advantage of this diversification. In the present research, we developed an ensemble model based on the three different complex network features previously described: weights; shortest path length and communicability. As voting scheme, we used a soft voting, which is based on averaging the probability scores given by the individual classifiers. Soft voting is usually preferred to hard voting (which is based on a simple majority voting rule), as it takes into account more information than only the binary prediction, i.e. the classifiers' uncertainty on the final decision they take.
As base learners, we used Multi-layer Perceptrons (MLPs) sharing the same hyper-parameters. Briefly speaking, an MLP is a feed-forward artificial neural network that can learn a nonlinear function approximator either for classification or regression (Murphy, 2018). In contrast to traditional logistic regression, which is based on a single weighted linear combination between the input layer and the output layer, an MLP provides one or more nonlinear (hidden) layers. In the present paper, we used an MLP with two hidden layers, 32 hidden units each; whereas, as activation function, we used the commonly used ReLU. Employing many more hidden layers would have had a negative impact on classification performance, given the disproportionately higher number of parameters to be optimized with respect to the number of training samples. Since the classification task is binary, the output layer performs a sigmoid activation:

$$
\operatorname{sigmoid}(x)=\frac{1}{1+e^{-x}},
$$

where $x$ is the feature vector from the preceding hidden layer. The network attempts to minimize a classic crossentropy loss function:

$$
\mathcal{H}(\theta)=\sum_{i=1}^{N} y_{i} \log \left(h_{\theta}\left(x_{i}\right)\right)+\left(1-y_{i}\right) \log \left(1-h_{\theta}\left(x_{i}\right)\right),
$$

where $\theta$ collectively indicates the parameters of the model, $N$ is the number of samples, and $y_{i}$ and $h_{\theta}\left(x_{i}\right)$ are the true and the predicted class label, respectively, for sample $x_{i}$. The network optimizes $\mathcal{H}(\theta)$ via backpropagation using the Limited-memory BFGS algorithm. This is an optimization algorithm in the family of quasi-Newton methods which is known to perform well when, as in our case, the data set is small (Morales and Nocedal, 2011). It is worth noting that, since the behavior of the neural network can be heavily influenced by different feature scales, features were normalized in the range $[0,1]$ before training.

We chose MLPs over other state-of-the-art classification algorithms mainly for two reasons. First, the use of nonlinearities within layers empowers the network with the capability of exploiting nonlinear relationships between data. Second, each layer can be equipped with a regularization term - the $\ell_{2}$ penalty in our casewhich can help mitigate overfitting in presence of highdimensional and possibly redundant features (Murphy. 


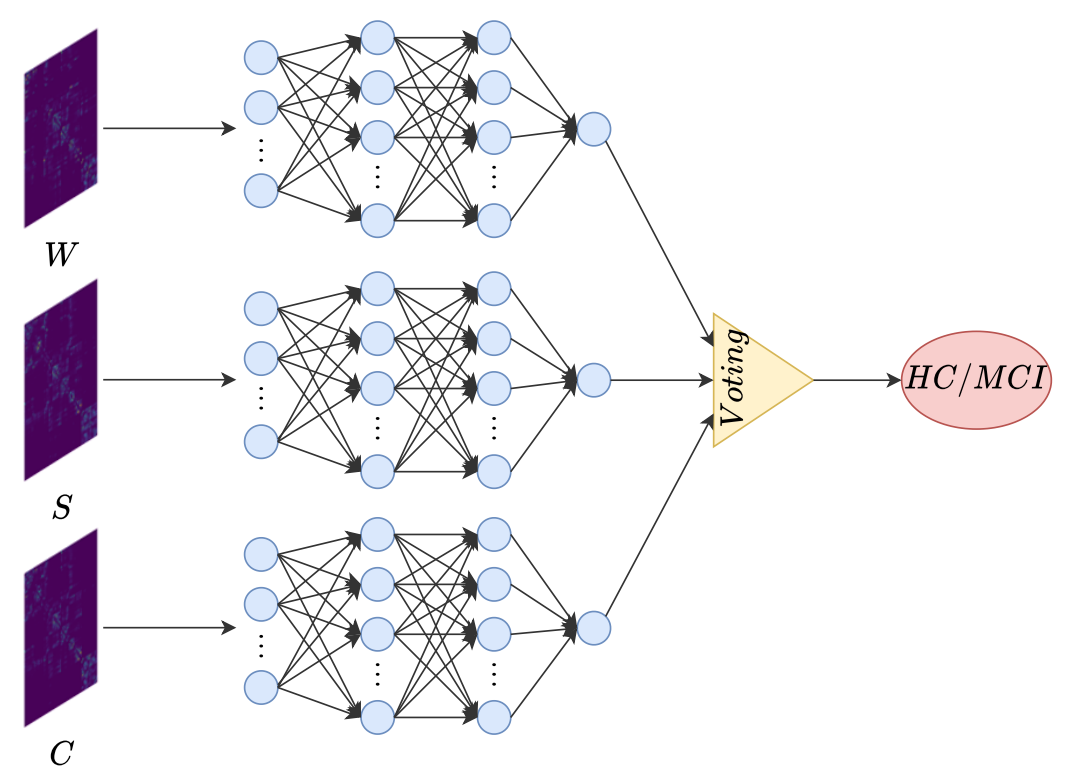

Figure 2: Proposed classification ensemble. The three complex network features are flattened and given as an input to three artificial neural networks sharing the same hyper-parameters. The final prediction is obtained as a soft voting of the individual classifiers' prediction. (Abbreviations: $W=$ weights; $S=$ shortest path length; $W=$ communicability; $H C=$ healthy controls; $M C I=$ mild cognitive impairment).

2018). The usefulness of neural network models in the clinical domain has been confirmed in several works, e.g. (Saniei et al. 2016) and (Diaz et al. 2019). An overall scheme of the proposed method is depicted in Fig. 2

\subsection{Balancing Strategies}

Most of machine learning methods are affected by the problem of having unbalanced data. As the imbalance increases, the classification models tend to favor the correct prediction of the instances in the over-represented class. In a diagnostic problem, this may bias a reliable estimate of the system's accuracy, as a higher number of samples in the pathological (or control) group may result in an overoptimistic estimate of sensitivity (or specificity). This is an acknowledged issue in the machine learning community, e.g. (Haixiang et al. 2017), and strategies to mitigate its effects for diagnostic purposes are sometimes adopted, e.g. (Duda et al. 2016) and (Angelillo et al. 2019).

In order to mitigate this issue, we employed three under-sampling approaches:

- Random under-sampling: this is the most naïve and easy way to under-sample the majority class. A sub- set sample of the over-represented class is selected randomly, then it is removed from the data set;

- Near miss-3: this belongs to the "near miss" family (Mani and Zhang, 2003) of methods which implements heuristics based on the $k$-nearest neighbors algorithm. The method proceeds in two steps: first, for each sample in the minority class, its $k$ nearest neighbors are detected; then, the examples in the majority class retained are the ones for which the average distance to the $k$ nearest neighbors is the largest. We set $k=3$. Near miss- 3 is known to be less susceptible to noise than the other variants;

- Instance hardness threshold: with this method, the MLP was trained and the subjects from the majority class for which the model returned the lower probability scores, i.e. those for which the model was less confident on the label to be assigned, were removed (Smith et al., 2014).

While random under-sampling does not use any criterion to adjust the class distribution, near miss and instance hardness threshold focus on the data points that may be 


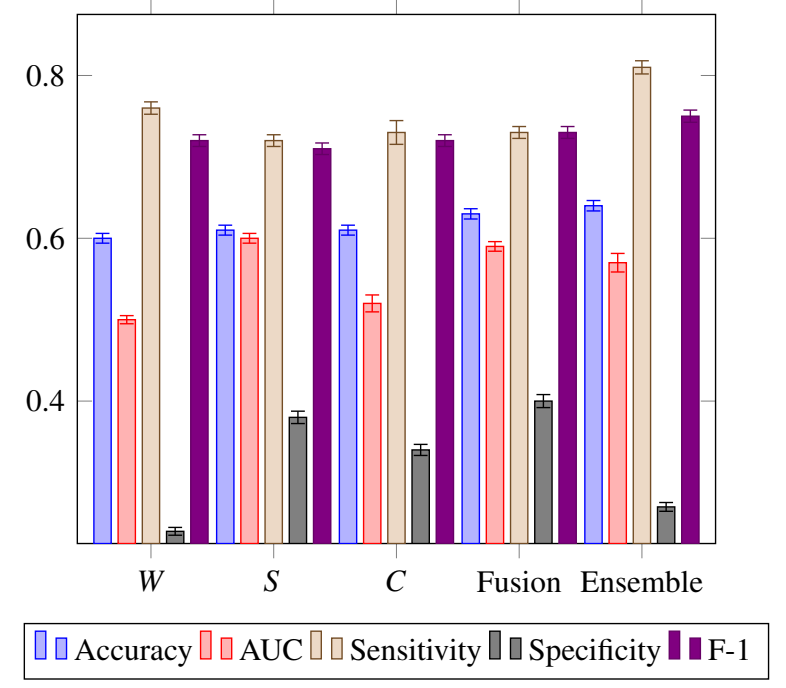

Figure 3: Classification performance on the unbalanced data. (Abbreviations: $W=$ weights; $S=$ shortest path length; $W=$ communicability).

harder to classify. This can introduce a bias which may lead to overoptimistic results.

\section{Experimental Results}

In this Section, we report the obtained results. As a baseline against which to compare the proposed method, we employed two approaches:

- The first baseline consisted in evaluating the predictive accuracy of the individual features when separately fed to the neural network model;

- The second baseline was based on combining the three feature vectors into a single high-dimensional vector fed to the neural network. This strategy represents a typical complementary approach to that based on the ensemble.

For a fair comparison, the classification models used for the baseline experiments shared the same hyperparameters of the MLP architecture used in the proposed ensemble.

Since the set of data is small, we validated the classification performance through a 10 -fold cross-validation. With this scheme, the set of data is partitioned into ten

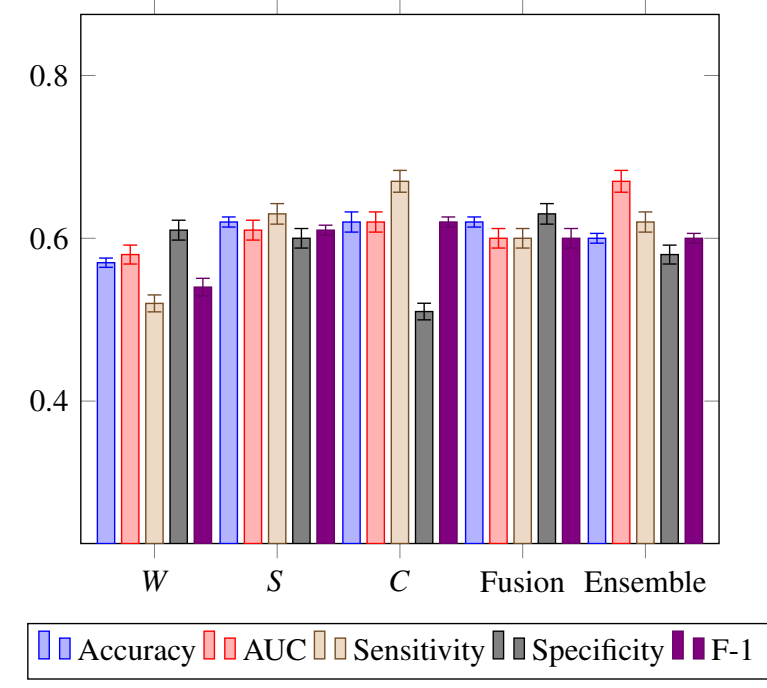

Figure 4: Classification performance after random under-sampling. (Abbreviations: $W=$ weights; $S=$ shortest path length; $W=$ communicability).

disjoint folds from which nine folds are used to train the learning algorithm, while the remaining fold is used to test it. This computation is iterated ten times, until each fold has been used as a test set once. In particular, we employed a stratified cross-validation, so that each fold contained roughly the same number of subjects from each diagnostic group. The entire procedure was repeated ten times, with different permutations of the training and test samples, for a better generalization of the performance.

As classification metrics, we used: accuracy; area under the ROC curve (AUC); sensitivity; specificity and F-1. We report the mean values of these metrics, averaged over all the cross-validation iterations. Also the standard errors are reported. In a clinical setting, sensitivity is one of the most important metrics to be monitored as it expresses the capability of the diagnostic tool to rule in disease when resulting in a positive response.

In the following, both the results obtained with the original unbalanced data and those obtained after undersampling are reported.

\subsection{Original Unbalanced Data}

Figure 3 shows the results obtained on the original unbalanced data (49 $\mathrm{HC}$ vs. $108 \mathrm{MCI}$ ). It can be observed 


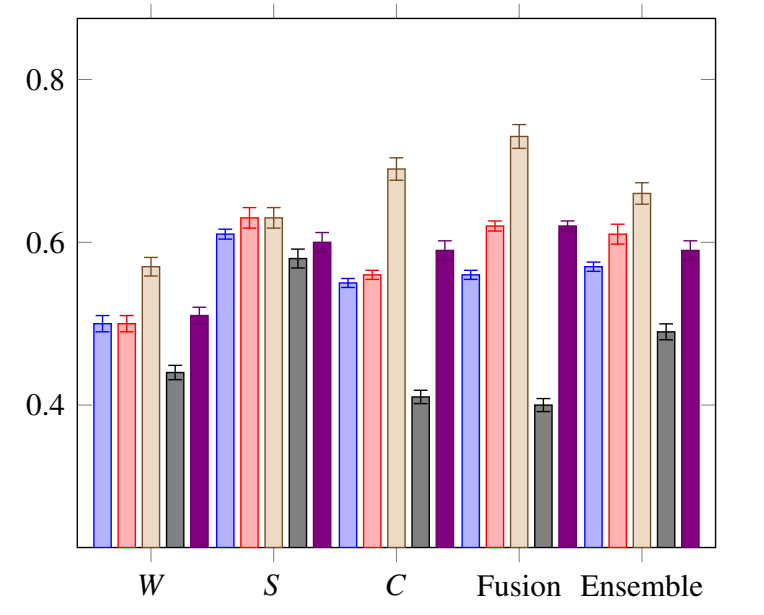

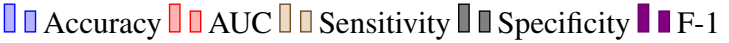

Figure 5: Classification performance after near miss under-sampling. (Abbreviations: $W=$ weights; $S=$ shortest path length; $W=$ communicability)

that the performance of the individual features are quite comparable. As expected, the proposed ensemble generally improved upon the performance of the individual features, achieving a sensitivity of $0.81 \pm 0.01$ and an F-1 of $0.75 \pm 0.01$. The fusion of features, instead, provided little or no improvement over the single network measures. The sensitivity obtained with the ensemble was found to be statistically significant different from the other classification strategies (Mann-Whitney U test at the significance level 0.01). The same applies to F-1, except for the comparison with the fusion of features $(p$-value $=0.014)$.

In general, very low values of specificity were obtained. As expected, this suggests that all classification models exhibited a preference for a more accurate prediction of the pathological group. This is confirmed by the lower values of AUC compared to sensitivity.

\subsection{Balanced Data}

In Fig. 4, the results obtained after randomly resampling the majority class are shown. An overall performance decrease can be observed. The best results were obtained by communicability (sensitivity $=0.67 \pm 0.02$ ) and the ensemble (AUC $=0.67 \pm 0.02$ ). These values were statistically significant different from the other classifica-

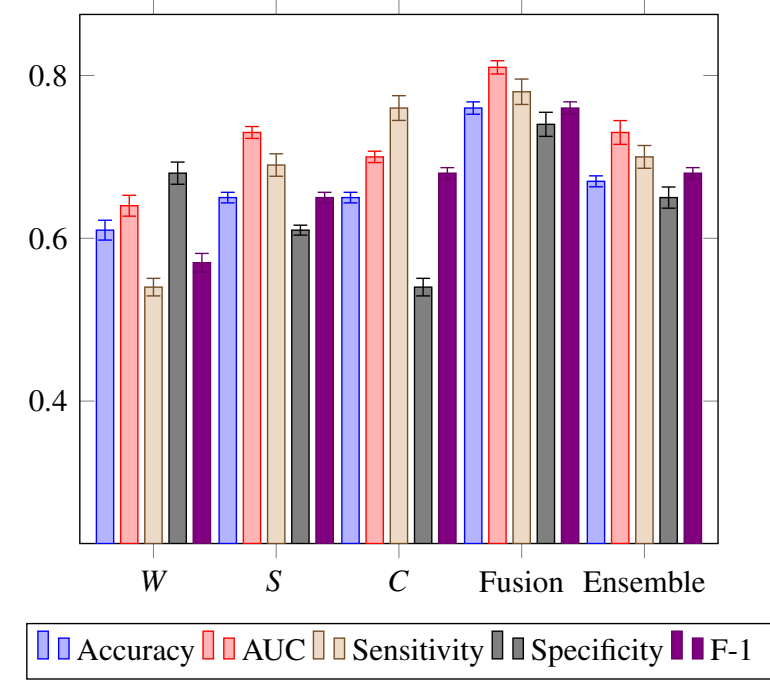

Figure 6: Classification performance after instance hardness threshold under-sampling. (Abbreviations: $W=$ weights; $S=$ shortest path length; $W=$ communicability).

tion strategies (Mann-Whitney $\mathrm{U}$ test at the significance level 0.05). Interestingly, shortest path length, communicability and the ensemble of features exhibited a sensitivity higher than specificity. This trend was reversed in the case of weights and the fusion of features.

When near miss under-sampling was used, some metrics slightly improved with respect to random undersampling. Once again, communicability provided the best sensitivity over the other individual features (i.e., a mean value of $0.69 \pm 0.02$ ). The ensemble and the fusion of features show similar accuracy and AUC; whereas, concerning the other metrics, a significantly $(p$-value $=0.009)$ higher sensitivity was achieved by the fusion of features (i.e., a mean value of $0.73 \pm 0.02$ ).

Finally, instance hardness threshold under-sampling provided an overall improvement over the other resampling techniques. This was expected, as this technique removes the data points that are harder to classify. Among the individual features, shortest path length and communicability provided the best results, with communicability achieving a sensitivity of $0.76 \pm 0.02$. The overall best performance, instead, were obtained by the fusion of features (i.e., a mean AUC of $0.81 \pm 0.01$ and a mean sensitivity of $0.78 \pm 0.02$ ). These performance were statistically sig- 
nificant different from the other classification strategies (Mann-Whitney U test at the significance level 0.01), except for sensitivity in the comparison with communicability ( $p$-value $=0.102$ ) and specificity in the comparison with weights $(p$-value $=0.032)$.

For all the three balanced data sets, although with performance lower compared to the unbalanced data, all classification strategies were almost always better in detecting the pathological condition in the pathological group. This highlights the robustness of the complex network approach in combination with the neural network paradigm against the type II error.

\section{Conclusion}

In this work, a novel classification method for MCI detection based on DWI data has been proposed. The method is based on ensembling neural network models fed with different graph measures. These measures can provide non-overlapping information on the same graphs under study, so the ensemble can benefit from this diversification. The proposed method exhibited good sensitivity either when using unbalanced or balanced groups. In fact, in this paper we have also shown the detrimental effects on classification performance when the pathological and the control group are equally represented.

Future works should attempt to identify the brain regions the connectivity of which is more related to the cognitive decline due to MCI. To this end, a feature importance analysis, based on the learning algorithm itself, could be done.

\section{Conflict of Interest}

The authors declare no conflict of interest.

\section{Acknowledgments}

Data collection and sharing for this project was funded by the Alzheimer's Disease Neuroimaging Initiative (ADNI) (National Institutes of Health Grant U01 AG024904) and DOD ADNI (Department of Defense award number W81XWH-12-2-0012). ADNI is funded by the National Institute on Aging, the National Institute of Biomedical Imaging and Bioengineering, and through generous contributions from the following: AbbVie, Alzheimer's Association; Alzheimer's Drug Discovery Foundation; Araclon Biotech; BioClinica, Inc.; Biogen; Bristol-Myers Squibb Company; CereSpir, Inc.; Cogstate; Eisai Inc.; Elan Pharmaceuticals, Inc.; Eli Lilly and Company; EuroImmun; F. Hoffmann-La Roche Ltd and its affiliated company Genentech, Inc.; Fujirebio; GE Healthcare; IXICO Ltd.; Janssen Alzheimer Immunotherapy Research \& Development, LLC.; Johnson \& Johnson Pharmaceutical Research \& Development LLC.; Lumosity; Lundbeck; Merck \& Co., Inc.; Meso Scale Diagnostics, LLC.; NeuroRx Research; Neurotrack Technologies; Novartis Pharmaceuticals Corporation; Pfizer Inc.; Piramal Imaging; Servier; Takeda Pharmaceutical Company; and Transition Therapeutics. The Canadian Institutes of Health Research is providing funds to support ADNI clinical sites in Canada. Private sector contributions are facilitated by the Foundation for the National Institutes of Health (www.fnih.org). The grantee organization is the Northern California Institute for Research and Education, and the study is coordinated by the Alzheimer's Therapeutic Research Institute at the University of Southern California. ADNI data are disseminated by the Laboratory for Neuro Imaging at the University of Southern California.

\section{References}

Amico, E., Goñi, J., 2018. Mapping hybrid functionalstructural connectivity traits in the human connectome. Network Neuroscience 2, 306-322.

Amlien, I., Fjell, A., 2014. Diffusion tensor imaging of white matter degeneration in Alzheimer's disease and mild cognitive impairment. Neuroscience 276, 206215.

Angelillo, M.T., Balducci, F., Impedovo, D., Pirlo, G., Vessio, G., 2019. Attentional pattern classification for automatic dementia detection. IEEE Access 7, 5770657716.

Crofts, J.J., Higham, D.J., 2009. A weighted communicability measure applied to complex brain networks. Journal of the Royal Society Interface 6, 411-414. 
Diaz, M., Ferrer, M.A., Impedovo, D., Pirlo, G., Vessio, G., 2019. Dynamically enhanced static handwriting representation for Parkinson's disease detection. Pattern Recognition Letters 128, 204-210.

Duda, M., Ma, R., Haber, N., Wall, D., 2016. Use of machine learning for behavioral distinction of autism and ADHD. Translational psychiatry 6, e732.

Ebadi, A., Dalboni da Rocha, J.L., Nagaraju, D.B., Tovar-Moll, F., Bramati, I., Coutinho, G., Sitaram, R., Rashidi, P., 2017. Ensemble classification of Alzheimer's disease and mild cognitive impairment based on complex graph measures from diffusion tensor images. Frontiers in neuroscience 11, 56.

Estrada, E., Hatano, N., 2008. Communicability in complex networks. Physical Review E 77, 036111.

Haixiang, G., Yijing, L., Shang, J., Mingyun, G., Yuanyue, H., Bing, G., 2017. Learning from classimbalanced data: Review of methods and applications. Expert Systems with Applications 73, 220-239.

Jeurissen, B., Tournier, J.D., Dhollander, T., Connelly, A., Sijbers, J., 2014. Multi-tissue constrained spherical deconvolution for improved analysis of multi-shell diffusion MRI data. NeuroImage 103, 411-426.

Lella, E., Amoroso, N., Diacono, D., Lombardi, A., Maggipinto, T., Monaco, A., Bellotti, R., Tangaro, S., 2019. Communicability characterization of structural DWI subcortical networks in Alzheimer's disease. Entropy 21,475 .

Lella, E., Amoroso, N., Lombardi, A., Maggipinto, T., Tangaro, S., Bellotti, R., 2018. Communicability disruption in Alzheimer's disease connectivity networks. Journal of Complex Networks 7, 83-100.

Lella, E., Estrada, E., 2020. Communicability distance reveals hidden patterns of Alzheimer's disease. Network Neuroscience, 1-23.

Lo, C.Y., Wang, P.N., Chou, K.H., Wang, J., He, Y., Lin, C.P., 2010. Diffusion tensor tractography reveals abnormal topological organization in structural cortical networks in Alzheimer's disease. Journal of Neuroscience 30, 16876-16885.
Mani, I., Zhang, I., 2003. kNN approach to unbalanced data distributions: a case study involving information extraction, in: Proceedings of workshop on learning from imbalanced datasets.

Morales, J.L., Nocedal, J., 2011. Remark on "algorithm 778: L-BFGS-B: Fortran subroutines for large-scale bound constrained optimization". ACM Trans. Math. Softw. 38, 7-1.

Murphy, K.P., 2018. Machine learning: A probabilistic perspective (adaptive computation and machine learning series).

Nir, T.M., Villalon-Reina, J.E., Prasad, G., Jahanshad, N., Joshi, S.H., Toga, A.W., Bernstein, M.A., Jack Jr, C.R., Weiner, M.W., Thompson, P.M., et al., 2015. Diffusion weighted imaging-based maximum density path analysis and classification of Alzheimer's disease. Neurobiology of aging 36, S132-S140.

Petersen, R.C., 2016. Mild cognitive impairment. CONTINUUM: Lifelong Learning in Neurology 22, 404.

Prasad, G., Joshi, S.H., Nir, T.M., Toga, A.W., Thompson, P.M., (ADNI, A.D.N.I., et al., 2015. Brain connectivity and novel network measures for Alzheimer's disease classification. Neurobiology of aging 36, S121-S131.

Reitz, C., Mayeux, R., 2014. Alzheimer disease: epidemiology, diagnostic criteria, risk factors and biomarkers. Biochemical pharmacology 88, 640-651.

Rolls, E.T., Joliot, M., Tzourio-Mazoyer, N., 2015. Implementation of a new parcellation of the orbitofrontal cortex in the automated anatomical labeling atlas. Neuroimage $122,1-5$.

Saniei, E., Setayeshi, S., Akbari, M.E., Navid, M., 2016. Parameter estimation of breast tumour using dynamic neural network from thermal pattern. Journal of Advanced Research 7, 1045-1055.

Schouten, T.M., Koini, M., de Vos, F., Seiler, S., de Rooij, M., Lechner, A., Schmidt, R., van den Heuvel, M., van der Grond, J., Rombouts, S.A., 2017. Individual classification of Alzheimer's disease with diffusion magnetic resonance imaging. Neuroimage 152, 476481 . 
Smith, M.R., Martinez, T., Giraud-Carrier, C., 2014. An instance level analysis of data complexity. Machine learning 95, 225-256.

Smith, R.E., Tournier, J.D., Calamante, F., Connelly, A., 2012. Anatomically-constrained tractography: improved diffusion MRI streamlines tractography through effective use of anatomical information. Neuroimage 62, 1924-1938.

Smith, R.E., Tournier, J.D., Calamante, F., Connelly, A., 2015a. The effects of SIFT on the reproducibility and biological accuracy of the structural connectome. Neuroimage 104, 253-265.

Smith, R.E., Tournier, J.D., Calamante, F., Connelly, A., 2015b. SIFT2: Enabling dense quantitative assessment of brain white matter connectivity using streamlines tractography. Neuroimage 119, 338-351.

Smith, S.M., 2002. Fast robust automated brain extraction. Human Brain Mapping 17, 143-155.

Theodoridis, S., 2015. Machine learning: a Bayesian and optimization perspective. Academic Press.

Tipnis, U., Amico, E., Ventresca, M., Goni, J., 2018. Modeling communication processes in the human connectome through cooperative learning. IEEE Transactions on Network Science and Engineering .

Tournier, J.D., Calamante, F., Connelly, A., 2010. Improved probabilistic streamlines tractography by 2 nd order integration over fibre orientation distributions, in: Proceedings of the International Society for Magnetic Resonance in Medicine, p. 1670.

Veraart, J., Novikov, D.S., Christiaens, D., Ades-Aron, B., Sijbers, J., Fieremans, E., 2016. Denoising of diffusion MRI using random matrix theory. NeuroImage 142, 394-406.

Vos, S.J., Verhey, F., Frölich, L., Kornhuber, J., Wiltfang, J., Maier, W., Peters, O., Rüther, E., Nobili, F., Morbelli, S., et al., 2015. Prevalence and prognosis of Alzheimer's disease at the mild cognitive impairment stage. Brain 138, 1327-1338.
Ward, A., Tardiff, S., Dye, C., Arrighi, H.M., 2013. Rate of conversion from prodromal Alzheimer's disease to Alzheimer's dementia: a systematic review of the literature. Dementia and geriatric cognitive disorders extra 3, 320-332.

Wee, C.Y., Yap, P.T., Li, W., Denny, K., Browndyke, J.N., Potter, G.G., Welsh-Bohmer, K.A., Wang, L., Shen, D., 2011. Enriched white matter connectivity networks for accurate identification of MCI patients. Neuroimage 54, 1812-1822.

Zhang, Y., Brady, M., Smith, S., 2001. Segmentation of brain MR images through a hidden markov random field model and the expectation-maximization algorithm. IEEE Transactions on Medical Imaging 20, 45-57. 